\title{
Distributed Filtering for Switched Nonlinear Positive Systems with Missing Measurements over Sensor Networks
}

\author{
Dong Wang, Zidong Wang, Guoyang Li and Wei Wang
}

\begin{abstract}
In this paper, the distributed filtering problem is investigated for a class of switched nonlinear positive systems over sensor networks. The randomly varying nonlinearities and missing measurements, which are governed by two mutually independent Bernoulli distributed white sequences, are taken into account. Based on the output measurements of the individual sensor and its neighbors, the distributed filter with positivity constraint is designed to ensure the prescribed average $l_{\infty}$ performance index of the estimation error dynamics. Special attention is paid to preserve the positivity of the underlying system as well as the sparseness of the addressed network topology. Sufficient conditions are established on the existence of the desired filters by using the linear programming approach and the filter gains are subsequently characterized. A simulation example is provided to illustrate the effectiveness of the proposed filtering method.
\end{abstract}

Index Terms-Switched systems, Positive systems, Distributed filtering, Stochastic nonlinearity, Missing measurements.

\section{INTRODUCTION}

It is widely known that many systems in the real world contain nonnegative states (e.g. population of animals and absolute temperature), and this gives rise to the so-called positive systems [1]. Mathematically, a positive system is such a system whose states and outputs are nonnegative as long as the initial conditions and inputs are nonnegative. Furthermore, a switched positive system is a dynamical system consisting of several positive subsystems with a switching signal, where the corresponding subsystem is activated during a certain interval. Such kind of systems has extensive applications in modelling practical systems such as networks employing transmission control protocol (TCP) [2] and rival mutation treatment dynamics [3].

Recently, there has been an increasing research interest in the control problems of switched positive systems in virtue of their significance in both theory and applications [4]-[10]. Several methods have been proposed to deal with the switching characteristics and positivity preservation, which include the average dwell time technique [5], the multiple Lyapunov

This work is in part supported by the National Natural Science Foundation of China (61104114, 61201035, 61374070, 61473055), Fundamental Research Funds for the Central Universities (DUT14QY14, DUT14QY31).

D. Wang, G. Li and W. Wang are with the School of Control Science and Engineering, Dalian University of Technology, Dalian 116024, P. R. China. (Email: dwang@dlut.edu.cn, liguoyang0418@qq.com,wangwei@dlut.edu.cn)

Z. Wang is with the Department of Computer Science, Brunel University London, Uxbridge, Middlesex, UB8 3PH, United Kingdom. He is also with the Faculty of Engineering, King Abdulaziz University, Jeddah 21589, Saudi Arabia. (Email: Zidong.Wang@brunel.ac.uk). function approach [4] as well as the switched Lyapunov function approach [6], etc. It is worth noticing that, in almost all relevant literature, the subsystems have been assumed to be linear. Nevertheless, in practical engineering, the nonlinearity is a ubiquitous yet complicated phenomenon that has been paid persistent research efforts in the past few decades [11]-[14]. It has been recently observed that the nonlinearities often occur in a probabilistic way especially in a networked environment where the network load is subject to random fluctuation. As such, the so-called randomly varying nonlinearities (RVNs) have drawn some initial research attention, see e.g. [15]-[20].

On the another research line, sensor networks have been gaining considerable research interest because of their wide application potentials in various areas such as environmental monitoring, multi-agent systems control [21]-[24] and wireless networks [25]-[27]. In a typical sensor network, the nodes are capable of sensing, computing and communicating. As one of important topics for information processing over sensor networks, the distributed filtering problem has recently attracted the attention from several research communities including communication, signal processing and control. Different from the traditional filtering applied to a single sensor only [28], in a sensor network, each sensor can receive the information from not only itself but also its neighbors according to the underlying network topology, and this makes the distributed nature of the filtering process. In this case, the key issue of the distributed filtering problem is how to effectively fuse the data from itself and its neighbors.

So far, several filtering algorithms have been proposed in the literature with respect to the sensor networks, see e.g. [29][37]. For example, in [31], the recovery problem of sparse signal in sensor networks has been solved by designing a regularized $H_{\infty}$ filter for each sensor node using the measurements distributed over the whole network. On the other hand, with the increasing scale of sensor networks, the crowd communication channel inevitably results in network-induced performance degradation of the designed distributed filters. One particular issue stemming from the imperfect communication among sensor nodes is the phenomenon of missing measurements (also called packet dropouts) that have been stirring recurring research interests during the past few years [38], [39]. A common approach to modelling missing measurements is to use a stochastic variable satisfying the Bernoulli distribution that takes values on either 1 or 0 , where 1 indicates the successful delivery and 0 represents missing measurements [16], [40]-[42]. 
It should be pointed out that, up to now, very little research effort has been made to investigate the distributed filter design problem for switched positive systems over lossy sensor networks in the presence of missing measurements, not to mention the case when the RVNs are also involved in the system. The main difficulties are threefold: 1) it is unclear how to evaluate the influence of the probabilistic missing measurements on the expected performance of the distributed filters; 2) it is pretty hard to deal with the positivity preservation and the sparseness of the network topology; and 3 ) it is fairly challenging to look into the impact from the RVNs on the convergence of the overall dynamics of the estimation errors. In order to overcome the difficulties identified above, in this paper, we are motivated to study the distributed filtering problem for a class of switched positive systems over lossy sensor networks, where the corresponding subsystem is subject to RVNs caused probably by environmental circumstances as well as missing measurements resulting from the network congestion among the communication nodes.

The main contributions of this paper are highlighted as follows: i) a novel average $l_{\infty}$ performance index is proposed to quantify the influences from both randomly occurring nonlinearities and missing measurements on the distributed filters, which is more appropriate than the conventional central $l_{\infty}$ performance criterion in the context of sensor networks; ii) special analysis techniques are developed to preserve both the positivity for system states and sparseness for network topology throughout the filtering process; iii) the impact of the introduced RVNs is examined on the switching dynamics among different subsystems in addition to the existing switching signals; and iv) a communication mechanism is adopted to update the filter input by using the data received at the last time-instant in case of the missing measurements.

This paper is organized as follows. In Section II, the switched positive systems with stochastic nonlinearity and the distributed filtering problem with missing measurements over sensor networks are introduced. Analysis and design of the distributed filter over sensor networks are carried out, respectively, in Section III and Section IV by using a linear programming technique. A simulation example is provided to verify the effectiveness of the presented results in Section V. Finally, conclusions are drawn in Section VI.

Notation. The notations used in this paper are fairly standard. The symbol $\otimes$ denotes the Kronecker product. $A \succeq 0$ $(\preceq, \succ, \prec)$ means that all entries of matrix $A$ are nonnegative (nonpositive, positive, negative). $\operatorname{diag}\{\ldots\}$ indicates a diagonal matrix. $1_{n}$ is $\left[\begin{array}{ccc}1 & \cdots & 1\end{array}\right]_{1 \times n}^{T} . R^{+}$means the set of the nonnegative real numbers. $l_{1}[0, \infty)$ is the space of summable infinite sequences with the norm $\|\omega\|_{1}=\sum_{k=0}^{\infty}\left|\omega_{i}\right|$.

\section{PROBLEM Formulation}

In this section, we introduce some preliminaries related to the distributed filtering problem and then introduce the problem under consideration.
Consider the discrete-time switched positive system with stochastic nonlinearities described as follows:

$$
\begin{aligned}
x(k+1) & =A_{\sigma(k)} x(k)+G_{\sigma(k)} \omega(k) \\
& +E_{\sigma(k)}(\beta(k) f(x(k))+(1-\beta(k)) g(x(k))), \\
z(k) & =M_{\sigma(k)} x(k)
\end{aligned}
$$

where $x(k) \in R_{n_{x}}$ is the state vector which cannot be measured directly; $z(k) \in R_{n_{z}}$ represents the output to be estimated; $\omega(k) \in R_{n_{\omega}}$ denotes the disturbance belonging to $l_{1}[0, \infty)$. The switching signal $\sigma(k)$, which denotes, for simplicity, $\sigma(k):[0, \infty) \rightarrow \Delta=\{1,2 \ldots N\}$, is an arbitrary switching sequence with $N$ being the number of subsystems. $A_{\sigma(k)}, E_{\sigma(k)}, G_{\sigma(k)}$ and $M_{\sigma(k)}$ are system matrices with appropriate dimensions. The nonlinear functions $f(x)$ and $g(x)$ (denoting $f(x(k))$ and $g(x(k))$ without confusion) are vectorvalued functions.

Assumption 1: The nonlinear functions $f(x)$ and $g(x)$ satisfy the following sector-bounded conditions

$$
\begin{aligned}
& \left(f(x)-U_{1} x\right)\left(f(x)-U_{2} x\right) \leq 0, \\
& \left(g(x)-U_{3} x\right)\left(g(x)-U_{4} x\right) \leq 0, \quad \forall x \in R_{n_{x}}^{+}
\end{aligned}
$$

where $U_{1}, U_{2}, U_{3}$ and $U_{4} \in R_{1 \times n}$ are real vectors.

Without loss of generality, supposing that $u_{1}=U_{1}-U_{2}$, $u_{2}=U_{3}-U_{4}$ are positive vectors, we can obtain

$$
\begin{aligned}
& \left(f(x)-U_{1} x\right) \leq 0, \quad\left(f(x)-U_{2} x\right) \geq 0, \\
& \left(g(x)-U_{3} x\right) \leq 0, \quad\left(g(x)-U_{4} x\right) \geq 0, \quad \forall x \in R_{n_{x}}^{+} .
\end{aligned}
$$

Remark 1: It is noted that the sector-bounded nonlinearity in Assumption 1 is universal in real systems. For instance, the amplifier has the phenomenon of saturation and the actuator could be with the dead zone. These can be represented by the sector-bounded nonlinearity.

Definition 1: [1] System (1) is said to be positive if, for the nonnegative initial condition $x(0), \omega(k), g(x), f(x)$ and $\sigma(k)$, the corresponding state trajectory $x(k) \geq 0$ and the estimated output $z(k) \geq 0$ hold for all $k \geq 0$.

Lemma 1: [1] System (1) is positive if and only if $A_{i} \succeq 0$, $E_{i} \succeq 0, G_{i} \succeq 0$ and $M_{i} \succeq 0, i \in \Delta$.

Remark 2: In this paper, the nonlinearities $f(x)$ and $g(x)$ are assumed to occur in a probabilistic way with an individual probability distribution that can be specified a prior through statistical tests. The concept of such RVNs was first introduced in [18] to reflect the random nature of the occurrence of the nonlinearities induced by network-load fluctuations within a networked environment.

The occurrence of the RVNs is governed by a stochastic variable $\beta(k)$ with

$$
\operatorname{Prob}\{\beta(k)=1\}=\bar{\beta}, \operatorname{Prob}\{\beta(k)=0\}=1-\bar{\beta}
$$

where $\bar{\beta} \in[0,1]$ is a known constant.

The measurements of system (1) are implemented by a sensor network. The communication topology of the sensor network consisting of $n$ sensor nodes is described by a fixed directed graph $G=(\nu, \varepsilon, A)$ of order $n$ with the set of nodes $\nu=\left\{1,2, \cdots, N_{s}\right\} . \varepsilon \subseteq \nu \times \nu$ is the set of edges and $A=\left[a_{i j}\right]$ is the weighted adjacency matrix with nonnegative adjacency 
element $a_{i j}$. An edge of $G$ is denoted by ordered pair $(i, j)$. A path from $i$ to $j$ is a sequence of the ordered edges of the form $(i, p),(p, q), \ldots,(r, j)$. If it is said that a directed graph is strongly connected, then, there is a directed path in the graph for any two distinct nodes $\mathrm{i}$ and $\mathrm{j}$. The adjacency elements associated with the edges of the graph are positive, i.e. $a_{i j}>0 \Leftrightarrow(i, j) \in \varepsilon$ which means that sensor $i$ can obtain information from sensor $j$. Moreover, we assume that $a_{i i}=1$ for all $i \in \nu$, and therefore $(i, i)$ can be regarded as an additional edge. The set of neighbors of node $i \in \nu$ plus the node itself are denoted by $N_{i}=\{j \in \nu:(i, j) \in \varepsilon\}$. The conditions on the network topology are quite general that 1) the graph is directed; and 2) the adjacency matrix is known.

The model for sensor $i, i \in \bar{N}=\left\{1, \cdots, N_{s}\right\}$ is

$$
y_{i}(k)=C_{i}(k) x(k)+D_{i}(k) \omega(k)
$$

where $y_{i}(k) \in R_{n_{y}}$ are the measurement output, $C_{i}$ and $D_{i}$ are known real matrices with appropriate dimensions.

In practice, the phenomena of missing measurements over sensor networks is often inevitable due to a variety of reasons such as resource constraints, intermittent sensor failures, network congestion and so on. Such a phenomenon can be described by a Bernoulli distributed sequence [16] as follows:

$$
\operatorname{Prob}\left\{\gamma_{i}(k)=1\right\}=\bar{\gamma}_{i}, \operatorname{Prob}\left\{\gamma_{i}(k)=0\right\}=1-\bar{\gamma}_{i}
$$

where $\bar{\gamma}_{i} \in[0,1]$ are known constants. If $y_{i}(k)$ are successfully sent to the filters, then $\gamma_{i}=1$, otherwise $\gamma_{i}=0$ and the last available measurement data is utilized. So, the received data is described by

$$
\begin{aligned}
\bar{y}_{i}(k) & =\gamma_{i}(k)\left[C_{i}(k) x(k)+D_{i}(k) \omega(k)\right] \\
& +\left(1-\gamma_{i}(k)\right) \bar{y}_{i}(k-1) .
\end{aligned}
$$

Assumption 2: The stochastic variables $\beta(k)$ and $\gamma_{i}(k)$ satisfy mutually independent Bernoulli distribution.

As is well known, an important point in designing distributed filters for a sensor network is how to fuse the information available from the sensor itself and its neighbors. Bearing this in mind, in this paper, we propose the following filter for sensor node $i$ :

$$
\begin{aligned}
\hat{x}_{i}(k+1) & =\sum_{j \in N_{i}} a_{i j} K_{i j} \hat{x}_{j}(k)+\sum_{j \in N_{i}} a_{i j} H_{i j} \bar{y}_{j}(k), \\
\hat{z}_{i}(k) & =M_{\sigma(k)} \hat{x}_{i}(k)
\end{aligned}
$$

where $\hat{x}_{i} \in R_{n_{x}}$ and $\hat{z}_{i} \in R_{n_{z}}$ are the estimation of the filter state and the estimation of the controlled output $z_{i}$ from the filter on sensor node $i$, respectively. Here, $K_{i j}$ and $H_{i j}$ are the filter gain matrices to be determined.

Let the filtering error be

$$
\tilde{z}_{i}(k):=z(k)-\hat{z}_{i}(k), \quad i=\bar{N} .
$$

Then, we obtain the following augmented system:

$$
\begin{aligned}
x(k+1) & =A_{\sigma(k)} x(k)+G_{\sigma(k)} \omega(k) \\
& +E_{\sigma(k)}\left[\begin{array}{ll}
\bar{\beta} & 1-\bar{\beta}
\end{array}\right]\left[\begin{array}{l}
f(x(k)) \\
g(x(k))
\end{array}\right] \\
& +(\beta(k)-\bar{\beta}) E_{\sigma(k)}\left[\begin{array}{ll}
1 & -1
\end{array}\right] \\
& \times
\end{aligned}
$$

$$
\begin{aligned}
\hat{x}_{i}(k+1) & =\sum_{j \in N_{i}} a_{i j} K_{i j} \hat{x}_{j}(k)-y_{j}(k-1) \\
& +\sum_{j \in N_{i}} \bar{\gamma}_{j} a_{i j} H_{i j} C_{j}(k) x(k) \\
& +\sum_{j \in N_{i}} \bar{\gamma}_{j} a_{i j} H_{i j} D_{j}(k) \omega(k) \\
& +\sum_{j \in N_{i}}\left(1-\bar{\gamma}_{j}\right) a_{i j} H_{i j} \bar{y}_{j}(k-1) \\
& +\sum_{j \in N_{i}}\left(\gamma_{j}(k)-\bar{\gamma}_{j}\right) a_{i j} H_{i j} C_{j}(k) x(k) \\
& +\sum_{j \in N_{i}}\left(\gamma_{j}(k)-\bar{\gamma}_{j}\right) a_{i j} H_{i j} D_{j}(k) \omega(k), \\
& =\bar{\gamma}_{i}\left(C_{i}(k) x(k)+D_{i}(k) \omega(k)\right) \\
& +\left(1-\bar{\gamma}_{i}\right) \bar{y}_{i}(k-1) \\
& +\left(\gamma_{i}(k)-\bar{\gamma}_{i}\right) C_{i}(k) x(k) \\
& +\left(\gamma_{i}(k)-\bar{\gamma}_{i}\right) D_{i}(k) \omega(k), \\
& =M_{\sigma(k)}\left(x(k)-\hat{x}_{i}(k)\right) . \\
\tilde{z}_{i}(k) &
\end{aligned}
$$

Definition 2: Given a disturbance attenuation level $\alpha>0$, the filtering error $\tilde{z}_{i}(k)$ from (9) is said to satisfy the average $l_{\infty}$ performance index if the following inequality holds:

$$
\frac{1}{N_{s}} \sum_{i=1}^{N_{s}} \sum_{k=1}^{\infty} E\left\{\left\|\tilde{z}_{i}(k)\right\|_{1}\right\}<\alpha \sum_{k=1}^{\infty}\|\omega(k)\|_{1} .
$$

Remark 3: The average $l_{\infty}$ performance index (10) over the $n$ sensors in a network is derived from the classical $l_{\infty}$ theory [16], which implies that the average energy gains from the disturbance to the average of all estimation errors should be less than the given disturbance attenuation level $\alpha$. Such an average $l_{\infty}$ performance index is more appropriate than the conventional central $l_{\infty}$ performance index in terms of quantifying the overall performance of the distributed filters.

Our objective in this paper is to design the filter gain matrices $K_{i j}$ and $H_{i j}$ such that the dynamics of the filtering errors $\tilde{z}_{i}(k)$ in (9) is asymptotically stable and also satisfies the average $l_{\infty}$ performance index (10) while preserving both the positivity for system states and sparseness for network topology throughout the filtering process.

\section{DISTRIBUTED FILTERS ANALYSIS}

In this section, the analysis results for the distributed filtering are provided for the discrete-time switched positive systems with stochastic nonlinearities over a sensor network plant. For the convenience of analysis, we define

$$
\begin{aligned}
& \eta(k)=\left[\begin{array}{lll}
\bar{x}^{T}(k) & \hat{x}^{T}(k) & y^{T}(k-1)
\end{array}\right]^{T}, \\
& \tilde{z}(k)=\bar{z}(k)-\hat{z}(k), \bar{x}(k)=1_{N_{s}} \otimes x(k), \\
& \hat{x}(k)=\left[\begin{array}{llll}
\hat{x}_{1}^{T}(k) & \hat{x}_{2}^{T}(k) & \cdots & \hat{x}_{N_{s}}^{T}(k)
\end{array}\right]^{T}, \\
& y(k)=\left[\begin{array}{llll}
y_{1}^{T}(k) & y_{2}^{T}(k) & \cdots & y_{N_{s}}^{T}(k)
\end{array}\right]^{T}, \\
& \hat{z}(k)=\left[\begin{array}{llll}
\hat{z}_{1}^{T}(k) & \hat{z}_{2}^{T}(k) & \cdots & \hat{z}_{N_{s}}^{T}(k)
\end{array}\right]^{T}, \\
& \Gamma(k)=\left[\begin{array}{ll}
F^{T}(\bar{x}(k)) & G^{T}(\bar{x}(k))
\end{array}\right]^{T},
\end{aligned}
$$




$$
\begin{aligned}
F(\bar{x}(k)) & =1_{N_{s}} \otimes f(x(k)), \\
G(\bar{x}(k)) & =1_{N_{s}} \otimes g(x(k)), \\
\bar{z}(k) & =1_{N_{s}} \otimes z(k), \bar{\omega}(k)=1_{N_{s}} \otimes \omega(k) .
\end{aligned}
$$

Then, we obtain the following augmented system:

$$
\begin{aligned}
\eta(k+1) & =\tilde{A}_{\sigma(k)} \eta(k)+\tilde{E}_{\sigma(k)} \Gamma(k)+\tilde{G}_{\sigma(k)} \bar{\omega}(k) \\
& +\tilde{A}_{\gamma \sigma(k)} \eta(k)+\tilde{E}_{\beta \sigma(k)} \Gamma(k)+\tilde{G}_{\gamma \sigma(k)} \bar{\omega}(k), \\
\tilde{z}(k) & =\tilde{M}_{\sigma(k)} \eta(k)
\end{aligned}
$$

where

$$
\begin{aligned}
& \tilde{A}_{\sigma(k)}=\left[\begin{array}{ccc}
\bar{A}_{\sigma(k)} & 0 & 0 \\
\bar{H} \bar{\Lambda}_{\gamma} \bar{C} & \bar{K} & \bar{H}\left(I-\bar{\Lambda}_{\gamma}\right) \\
\bar{\Lambda}_{\gamma} \bar{C} & 0 & I-\bar{\Lambda}_{\gamma}
\end{array}\right], \\
& \bar{A}_{\sigma(k)}=I_{N_{s}} \otimes A_{\sigma(k)}, \bar{\Lambda}_{\gamma}=\operatorname{diag}\left\{\bar{\gamma}_{1}, \bar{\gamma}_{2}, \ldots, \bar{\gamma}_{N_{s}}\right\}, \\
& \bar{K}=\left[\bar{K}_{i j}\right]_{N_{s} n_{x} \times N_{s} n_{x}}, \bar{H}=\left[\bar{H}_{i j}\right]_{N_{s} n_{x} \times N_{s} n_{y}}, \\
& \bar{H}_{i j}=\left\{\begin{array}{c}
a_{i j} H_{i j}, i=1,2, \ldots, N_{s}, j \in N_{i} \\
0, i=1,2, \ldots, N_{s}, j \notin N_{i}
\end{array},\right. \\
& \bar{K}_{i j}=\left\{\begin{array}{c}
a_{i j} K_{i j}, i=1,2, \ldots, N_{s}, j \in N_{i} \\
0, i=1,2, \ldots, N_{s}, j \notin N_{i}
\end{array},\right. \\
& \bar{C}=\operatorname{diag}\left\{C_{1}, C_{2}, \ldots, C_{N_{s}}\right\}, \\
& \tilde{E}_{\sigma(k)}=\left[\begin{array}{cc}
\bar{E}_{\sigma(k)} \bar{\Lambda}_{\beta} & \bar{E}_{\sigma(k)}\left(I-\bar{\Lambda}_{\beta}\right) \\
0 & 0 \\
0 & 0
\end{array}\right] \text {, } \\
& \bar{E}_{\sigma(k)}=I_{N_{s}} \otimes E_{\sigma(k)}, \bar{\Lambda}_{\beta}=I_{N_{s}} \otimes \bar{\beta}, \bar{G}_{\sigma(k)}=I_{N_{s}} \otimes G_{\sigma(k)}, \\
& \bar{D}=\operatorname{diag}\left\{D_{1}, D_{2}, \ldots, D_{N_{s}}\right\} \text {, } \\
& \tilde{A}_{\gamma \sigma(k)}=\left[\begin{array}{ccc}
0 & 0 & 0 \\
\bar{H}\left(\Lambda_{\gamma}-\bar{\Lambda}_{\gamma}\right) \bar{C} & 0 & -\bar{H}\left(\Lambda_{\gamma}-\bar{\Lambda}_{\gamma}\right) \\
\left(\Lambda_{\gamma}-\bar{\Lambda}_{\gamma}\right) \bar{C} & 0 & -\left(\Lambda_{\gamma}-\bar{\Lambda}_{\gamma}\right)
\end{array}\right] \text {, } \\
& \Lambda_{\gamma}=\operatorname{diag}\left\{\gamma_{1}(k), \gamma_{2}(k), \ldots, \gamma_{N s}(k)\right\} \text {, } \\
& \tilde{E}_{\beta \sigma(k)}=(\beta(k)-\bar{\beta})\left[\begin{array}{cc}
\bar{E}_{\sigma(k)} & -\bar{E}_{\sigma(k)} \\
0 & 0 \\
0 & 0
\end{array}\right] \text {, } \\
& \tilde{G}_{\gamma \sigma(k)}=\left[\begin{array}{c}
0 \\
\bar{H}\left(\Lambda_{\gamma}-\bar{\Lambda}_{\gamma}\right) \bar{D} \\
\left(\Lambda_{\gamma}-\bar{\Lambda}_{\gamma}\right) \bar{D}
\end{array}\right], \tilde{G}_{\sigma(k)}=\left[\begin{array}{c}
\bar{G}_{\sigma(k)} \\
\bar{H} \bar{\Lambda}_{\gamma} \bar{D} \\
\bar{\Lambda}_{\gamma} \bar{D}
\end{array}\right], \\
& \tilde{M}_{\sigma(k)}=\left[\begin{array}{lll}
\bar{M}_{\sigma(k)} & -\bar{M}_{\sigma(k)} & 0
\end{array}\right] \text {, } \\
& \bar{M}_{\sigma(k)}=I_{N_{s}} \otimes M_{\sigma(k)} \text {. }
\end{aligned}
$$

Based on the transformation conducted above, the following theorem is provided to ensure the stability of the augmented system.

Theorem 1: Let the scalars $\alpha>0, \bar{\beta} \in[0,1]$ and $\bar{\gamma}_{i} \in[0,1]$ $\left(i \in\left\{1,2, \cdots, N_{s}\right\}\right)$ be given. System (11) is asymptotically stable and satisfies the performance specification (10) if there exist vectors $P_{i} \succeq 0(i, j \in \Delta)$ such that

$$
\begin{aligned}
& \tilde{A}_{i}^{T} P_{j}-P_{i}+2 \tilde{Y}_{1}^{T} 1_{N_{s}\left(2 n_{x}+n_{y}\right)} \\
&-\tilde{Y}_{2}^{T} 1_{N_{s}\left(2 n_{x}+n_{y}\right)}+\vec{M}_{i}^{T} 1_{N_{s}\left(2 n_{x}+n_{y}\right)} \preceq 0, \\
& \tilde{E}_{i}^{T} P_{j}-1_{2 N_{s}} \preceq 0, \tilde{G}_{i}^{T} P_{j}-\alpha 1_{N_{s} n_{\omega}} \preceq 0
\end{aligned}
$$

where

$$
\begin{aligned}
\tilde{Y}_{1} & =\left[\begin{array}{ccc}
\bar{Y}_{1}^{T} & 0 & 0
\end{array}\right]^{T}, \tilde{Y}_{2}=\left[\begin{array}{ccc}
\bar{Y}_{2}^{T} & 0 & 0
\end{array}\right]^{T}, \\
\vec{M}_{\sigma(k)} & =\operatorname{diag}\left\{\bar{M}_{\sigma(k)}, \bar{M}_{\sigma(k)}, 0\right\} .
\end{aligned}
$$

proof 1: Select the following co-positive Lyapunov function

$$
V(k)=\eta^{T}(k) P_{\sigma(k)}
$$

where $P_{\sigma(k)}$ are positive vectors. Then, the mathematical expectation of Lyapunov function (13) is

$$
\begin{aligned}
& E\{V(k+1) \mid V(k)\} \\
& =\left(\tilde{A}_{\sigma(k)} \eta(k)+\tilde{E}_{\sigma(k)} \Gamma(k)+\tilde{G}_{\sigma(k)} \bar{\omega}(k)\right)^{T} P_{\sigma(k+1)} .
\end{aligned}
$$

Using switched Lyapunov function method and calculating the forward difference of (13) along the trajectories of system (11) lead to

$$
\begin{aligned}
E(\Delta V(k)) & =E\left\{\eta^{T}(k+1) P_{\sigma(k+1)}-\eta^{T}(k) P_{\sigma(k)}\right\} \\
& =\left(\bar{A}_{\sigma(k)} \eta(k)+\tilde{E}_{\sigma(k)} \Gamma(k)\right)^{T} P_{\sigma(k+1)} \\
& +\left(\tilde{G}_{\sigma(k)} \bar{\omega}(k)\right)^{T} P_{\sigma(k+1)}-\eta^{T}(k) P_{\sigma(k)} \\
& =\eta^{T}(k)\left(\bar{A}_{\sigma(k)}^{T} P_{\sigma(k+1)}-P_{\sigma(k)}\right)+\Gamma^{T}(k) \\
& \times \tilde{E}_{\sigma(k)}^{T} P_{\sigma(k+1)}+\bar{\omega}^{T}(k) \tilde{G}_{\sigma(k)}^{T} P_{\sigma(k+1)} \cdot(14)
\end{aligned}
$$

From (2), it is seen that

$$
\begin{gathered}
\left(F(\bar{x}(k))-\bar{U}_{1} \bar{x}(k)\right)^{T}\left(F(\bar{x}(k))-\bar{U}_{2} \bar{x}(k)\right) \leq 0, \\
\left(G(\bar{x}(k))-\bar{U}_{3} \bar{x}(k)\right)^{T}\left(G(\bar{x}(k))-\bar{U}_{4} \bar{x}(k)\right) \leq 0
\end{gathered}
$$

where

$$
\begin{aligned}
& \bar{U}_{1}=I_{N_{s}} \otimes U_{1}, \quad \bar{U}_{2}=I_{N_{s}} \otimes U_{2}, \\
& \bar{U}_{3}=I_{N_{s}} \otimes U_{3}, \quad \bar{U}_{4}=I_{N_{s}} \otimes U_{4} .
\end{aligned}
$$

Hence, it can be obtained that

$$
\left(\Gamma(k)-\bar{Y}_{1} \bar{x}(k)\right)^{T}\left(\Gamma(k)-\bar{Y}_{2} \bar{x}(k)\right) \leq 0
$$

where

$$
\bar{Y}_{1}=\left[\begin{array}{cc}
\bar{U}_{1}^{T} & \bar{U}_{3}^{T}
\end{array}\right]^{T}, \bar{Y}_{2}=\left[\begin{array}{cc}
\bar{U}_{2}^{T} & \bar{U}_{4}^{T}
\end{array}\right]^{T} .
$$

Furthermore, we can get

$$
\begin{aligned}
& E\{\Delta V(k)\} \\
< & \eta^{T}(k)\left(\tilde{A}_{\sigma(k)}^{T} P_{\sigma(k+1)}-P_{\sigma(k)}\right)+\Gamma^{T}(k)\left(\widetilde{E}_{\sigma(k)}^{T} P_{\sigma(k+1)}\right. \\
+ & \bar{\omega}^{T}(k) \tilde{G}_{\sigma(k)}^{T} P_{\sigma(k+1)}-2\left(\Gamma(k)-\bar{Y}_{1} \bar{x}(k)\right)^{T} 1_{2 N_{s}} \\
+ & \left(\Gamma(k)-\bar{Y}_{2} \bar{x}(k)\right)^{T} 1_{2 N_{s}} \\
= & \eta^{T}(k)\left(\tilde{A}_{\sigma(k)}^{T} P_{\sigma(k+1)}-P_{\sigma(k)}\right)-\eta^{T}(k) \tilde{Y}_{2}^{T} 1_{N_{s}\left(2 n_{x}+n_{y}\right)} \\
+ & 2 \eta^{T}(k) \tilde{Y}_{1}^{T} 1_{N_{s}\left(2 n_{x}+n_{y}\right)}+\bar{\omega}^{T}(k) \tilde{G}_{\sigma(k)}^{T} P_{\sigma(k+1)} \\
+ & \Gamma^{T}(k)\left(\tilde{E}_{\sigma(k)}^{T} P_{\sigma(k+1)}-1_{2 N_{s}}\right) .
\end{aligned}
$$

It can be seen from (16) that $E\{\Delta V(k)\}<0$ if $\omega(k)=0$ and

$$
\begin{aligned}
\tilde{A}_{\sigma(k)}^{T} P_{\sigma(k+1)}-P_{\sigma(k)} & \\
+2 \widetilde{Y}_{1}^{T} 1_{N_{s}\left(2 n_{x}+n_{y}\right)}-\tilde{Y}_{2}^{T} 1_{N_{s}\left(2 n_{x}+n_{y}\right)} & \preceq 0, \\
\tilde{E}_{\sigma(k)}^{T} P_{\sigma(k+1)}-1_{2 N_{s}} & \preceq 0
\end{aligned}
$$

which implies that system (11) is asymptotically stable in the absence of the disturbances if (17) holds. Without loss of generality, letting $\sigma_{k}=i$ and $\sigma_{k+1}=j$, (17) is equivalent to the first two inequalities of (12). 
The following performance index is defined to obtain average $l_{\infty}$ performance of system (11):

$$
J=\frac{1}{N_{s}} \sum_{i=1}^{N_{s}} \sum_{k=1}^{\infty} E\left\{\left\|\tilde{z}_{i}(k)\right\|_{1}\right\}-\alpha \sum_{k=1}^{\infty}\|\omega(k)\|_{1} .
$$

By making use of the property of the norm, it is obtained that

$$
\begin{aligned}
\left\|\tilde{z}_{i}(k)\right\|_{1} & =\left\|M_{\sigma(k)}\left(x(k)-\hat{x}_{i}(k)\right)\right\|_{1} \\
& <\left\|M_{\sigma(k)} x(k)\right\|_{1}+\left\|M_{\sigma(k)} \hat{x}_{i}(k)\right\|_{1} \\
& =x^{T}(k) M_{\sigma(k)}^{T} 1_{n_{x}}+\hat{x}_{i}^{T}(k) M_{\sigma(k)}^{T} 1_{n_{x}} .
\end{aligned}
$$

Furthermore, combining with (11) leads to

$$
\begin{aligned}
J & =\frac{1}{N_{s}} \sum_{i=1}^{N_{s}} \sum_{k=1}^{\infty} E\left\{\left\|\tilde{z}_{i}(k)\right\|_{1}\right\}-\alpha \sum_{k=1}^{\infty}\|\omega(k)\|_{1} \\
& =\sum_{k=1}^{\infty}\left\{\frac{1}{N_{s}} \sum_{i=1}^{N_{s}} E\left\{\left\|\tilde{z}_{i}(k)\right\|_{1}\right\}-\alpha\|\omega(k)\|_{1}\right\} \\
& <\sum_{k=1}^{\infty}\left\{\frac{1}{N_{s}} \sum_{i=1}^{N_{s}} E\left\{x^{T}(k) M_{\sigma(k)}^{T} 1_{n_{x}}\right\}\right. \\
& \left.+\frac{1}{N_{s}} \sum_{i=1}^{N_{s}} E\left\{\hat{x}_{i}^{T}(k) M_{\sigma(k)}^{T} 1_{n_{x}}\right\}-\alpha \omega^{T}(k) 1_{n_{w}}\right\} \\
& =\sum_{k=1}^{\infty} \frac{1}{N_{s}}\left\{\eta^{T}(k) \vec{M}_{\sigma(k)}^{T} 1_{N_{s}\left(2 n_{x}+n_{y}\right)}\right. \\
& \left.-\alpha \bar{\omega}^{T}(k) 1_{N_{s} n_{\omega}}\right\} .
\end{aligned}
$$

Based on the zero-initial conditions $\eta(0)^{T}=0$ and $V(k) \geq 0$ when $k \geq 0$, we have

$$
\begin{aligned}
J & =\sum_{k=1}^{\infty} \frac{1}{N_{s}}\left\{\eta^{T}(k) \vec{M}_{\sigma(k)}^{T} 1_{N_{s}\left(2 n_{x}+n_{y}\right)}-\alpha \bar{\omega}^{T}(k) 1_{N_{s} n_{\omega}}\right. \\
& +\Delta V(k)-\Delta V(k)\} \\
& \leq \sum_{k=1}^{\infty} \frac{1}{N_{s}}\left\{\eta^{T}(k) \vec{M}_{\sigma(k)}^{T} 1_{N_{s}\left(2 n_{x}+n_{y}\right)}-\alpha \bar{\omega}^{T}(k) 1_{N_{s} n_{\omega}}\right. \\
& +\eta^{T}(k)\left(\tilde{A}_{\sigma(k)}^{T} P_{\sigma(k+1)}-P_{\sigma(k)}+2 \tilde{Y}_{1}^{T} 1_{N_{s}\left(2 n_{x}+n_{y}\right)}\right. \\
& \left.-\tilde{Y}_{2}^{T} 1_{N_{s}\left(2 n_{x}+n_{y}\right)}\right)+\Gamma^{T}(k)\left(\tilde{E}_{\sigma(k)}^{T} P_{\sigma(k+1)}-1_{2 N_{s}}\right) \\
& \left.+\bar{\omega}^{T}(k) \tilde{G}_{\sigma(k)}^{T} P_{\sigma(k+1)}\right\} \\
& =\sum_{k=1}^{\infty} \frac{1}{N_{s}}\left\{\eta ^ { T } ( k ) \left(\tilde{A}_{\sigma(k)}^{T} P_{\sigma(k+1)}-P_{\sigma(k)}\right.\right. \\
& +2 \tilde{Y}_{1}^{T} 1_{N_{s}\left(2 n_{x}+n_{y}\right)}-\tilde{Y}_{2}^{T} 1_{N_{s}\left(2 n_{x}+n_{y}\right)} \\
& \left.+\vec{M}_{\sigma(k)}^{T} 1_{N_{s}\left(2 n_{x}+n_{y}\right)}\right)+\Gamma^{T}(k)\left(\tilde{E}_{\sigma(k)}^{T} P_{\sigma(k+1)}-1_{2 N_{s}}\right) \\
& \left.+\bar{\omega}^{T}(k)\left(\tilde{G}_{\sigma(k)}^{T} P_{\sigma(k+1)}-\alpha 1_{N_{s} n_{\omega}}\right)\right\} \\
& =\sum_{k=1}^{\infty} \frac{1}{N_{s}}\left(\tilde{\eta}^{T}(k) \Xi_{k, k+1}\right)
\end{aligned}
$$

where

$$
\begin{aligned}
& \tilde{\eta}(k)=\left[\begin{array}{lll}
\eta^{T}(k) & \Gamma^{T}(k) & \bar{\omega}^{T}(k)
\end{array}\right]^{T}, \\
& \Xi_{k, k+1}=\left[\begin{array}{c}
\Pi(k), \\
\tilde{E}_{\sigma(k)}^{T} P_{\sigma(k+1)}-1_{2 N_{s}} \\
\tilde{G}_{\sigma(k)}^{T} P_{\sigma(k+1)}-\alpha 1_{N_{s} n_{\omega}}
\end{array}\right] \text {, } \\
& \Pi(k)=\tilde{A}_{\sigma(k)}^{T} P_{\sigma(k+1)}-P_{\sigma(k)}+2 \tilde{Y}_{1}^{T} 1_{N_{s}\left(2 n_{x}+n_{y}\right)} \\
& -\tilde{Y}_{2}^{T} 1_{N_{s}\left(2 n_{x}+n_{y}\right)}+\tilde{M}_{\sigma(k)}^{T} 1_{N_{s}\left(2 n_{x}+n_{y}\right)} \text {. }
\end{aligned}
$$

Obviously, $J \leq 0$ holds if $\Xi_{k, k+1} \preceq 0$. Letting $\sigma_{k}=i$ and $\sigma_{k+1}=j$ based on $\Xi_{k, k+1}$, we can get the inequality (12). This means that if (12) holds, system (11) is asymptotically stable and also satisfies the performance index (10). The proof is now complete.

Remark 4: In (14), because of the stringent requirements that $\tilde{E}_{\sigma(k)}^{T} P_{\sigma(k+1)}$ and $P_{\sigma(k)}$ are positive vectors, it might not be easy to ensure $E\{\Delta V(k)\}<0$. To deal with such an issue, we take into account the sector-bounded condition in (15) and introduce $2 \tilde{Y}_{1}^{T} 1_{N_{s}\left(2 n_{x}+n_{y}\right)}-\tilde{Y}_{2}^{T} 1_{N_{s}\left(2 n_{x}+n_{y}\right)}$ associated with the nonlinear function $\Gamma^{T}(x)$. This way, some auxiliary vectors are added which would help enhance the feasibility of the matrix inequalities (12).

Remark 5: It should be pointed out that, based on the widely accepted $H_{\infty}$ index, the average $H_{\infty}$ performance index has been used in existing literature (e.g. [16], [39]) for the distributed filter design over the sensor network, where the disturbances belongs to the $l_{2}$ space and the Lyapunov function with the quadratic form is constructed. However, in our paper, because of the nonnegative property of switched positive systems, a straightforward application of distributed filter design for non-positive dynamical systems might be infeasible. Instead, an average $l_{\infty}$ index is introduced for the design of distributed filter and the co-positive Lyapunov function is constructed, which preserve the positivity in the filtering procedure.

\section{Distributed FILTER DESIGN}

We are now in a position to present sufficient conditions on the existence of the distributed filter and characterize the filter gains.

Theorem 2: For system (1), let the scalars $\alpha>0, \bar{\beta} \in[0,1]$ and $\bar{\gamma}_{i} \in[0,1]\left(i \in\left\{1,2, \cdots, N_{s}\right\}\right)$ be given. The distributed filter (8) with missing measurements (7) can be designed to ensure the asymptotic stability of the filtering error dynamics and also achieve the average $l_{\infty}$ performance index (10) if there exists positive matrices $K_{s} \in R_{N_{s} n_{x} \times N_{s} n_{x}}^{+}, H_{s} \in$ $R_{N_{s} n_{x} \times N_{s} n_{y}}^{+}, p_{i 1} \in R_{N_{s} n_{x} \times 1}^{+}, q \in R_{N_{s} n_{x} \times 1}^{+}, p_{i 3} \in R_{N_{s} n_{y} \times 1}^{+}$, $(i, j \in \Delta)$ such that the following inequalities hold:

$$
\begin{aligned}
& \bar{A}_{i}^{T} p_{j 1}+\left(\bar{\Lambda}_{\gamma} \bar{C}\right)^{T} H_{s}^{T} 1_{N_{s} n_{x}}+\left(\bar{\Lambda}_{\gamma} \bar{C}\right)^{T} p_{j 3} \\
&-p_{i 1}+2 \bar{Y}_{1}^{T} 1_{2 N_{s}}-\bar{Y}_{2}^{T} 1_{2 N_{s}}+\bar{M}_{i}^{T} 1_{N_{s} n_{x}} \preceq 0, \\
& \bar{G}_{i}^{T} p_{j 1}+\left(\bar{\Lambda}_{\gamma} \bar{D}\right)^{T} H_{s}^{T} 1_{N_{s} n_{x}} \\
&-\left(\bar{\Lambda}_{\gamma} \bar{D}\right)^{T} p_{j 3}-\alpha 1_{N_{s} n_{\omega}} \preceq 0,
\end{aligned}
$$




$$
\begin{aligned}
K_{s}^{T} 1_{N_{s} n_{x}}-q+\bar{M}_{i}^{T} 1_{N_{s} n_{x}} & \preceq 0, \\
\left(I-\bar{\Lambda}_{\gamma}\right)^{T} H_{s}^{T} 1_{N_{s} n_{x}}+\left(I-\bar{\Lambda}_{\gamma}\right)^{T} p_{j 3}-p_{i 3} & \preceq 0, \\
\bar{\beta} \bar{E}_{i}^{T} p_{j 1}-1_{N_{s}} \preceq 0, \quad(1-\bar{\beta}) \bar{E}_{i}^{T} p_{j 1}-1_{N_{s}} & \preceq 0(18)
\end{aligned}
$$

where

$$
\begin{gathered}
K_{s}=\left[K_{s i j}\right]_{N_{s} n_{x} \times N_{s} n_{x}}, H_{s}=\left[H_{s i j}\right]_{N_{s} n_{x} \times N_{s} n_{y},} \\
H_{s i j}=\left\{\begin{array}{c}
H_{s i j}, j \in N_{i} \\
0, j \notin N_{i}
\end{array}, K_{s i j}=\left\{\begin{array}{c}
K_{s i j}, j \in N_{i} \\
0, j \notin N_{i}
\end{array} .\right.\right.
\end{gathered}
$$

Moreover, if there exists a feasible solution to (18), then the desired filter gain can be determined by

$$
\bar{H}^{T}=H_{s}^{T} q_{d}^{-1}, \bar{K}^{T}=K_{s}^{T} q_{d}^{-1}
$$

where $q_{d}=\operatorname{diag}\left\{q_{1}, q_{2}, \cdots, q_{N_{s} n_{x}}\right\}, q_{i}, i=1, \cdots, N_{s} n_{x}$ are the elements of the vector $q$.

proof 2: Defining

$$
\begin{aligned}
H_{s}^{T} & =\bar{H}^{T} q_{d}, K_{s}^{T}=\bar{K}^{T} q_{d}, \\
P_{i} & =\left[\begin{array}{lll}
p_{i 1}^{T} & q^{T} & p_{i 3}^{T}
\end{array}\right]^{T}
\end{aligned}
$$

and substituting (20) into (18), we can readily get (12). In other words, if there exist positive matrices $K_{s} \in R_{N_{s} n_{x} \times N_{s} n_{x}}^{+}$, $H_{s} \in R_{N_{s} n_{x} \times N_{s} n_{y}}^{+}, p_{i 1} \in R_{N_{s} n_{x} \times 1}^{+}, q \in R_{N_{s} n_{x} \times 1}^{+}$, $p_{i 3} \in R_{N_{s} n_{y} \times 1}^{+},(i, j \in \Delta)$ such that (18) are satisfied, then system (11) is asymptotically stable and satisfies performance specifications (10), which means system (11) is insensitive against the disturbance by means of the index $\alpha$. This means that the design problem of distributed filters (8) for system (1) with measurement missing (7) is solvable. At last, by using (20), the filter gains are constructed by (19). The proof is now complete.

Remark 6: It is observed from (19) that there are the proportion $q_{d}$ between $H_{s}$ and $\bar{H}$ as well as $K_{s}$ and $\bar{K}$ in Theorem 2. Note that the vector $q$ in (18) is transformed into the product of a diagonal matrix $q_{d}$ whose elements are those of $q$ and a vector $1_{N_{s} n_{x}}$ whose elements are all ones. Such matrix transformation technique is proposed to deal with the challenges resulting from the sparseness of the sensor network topology and preserving structure of the distributed filters.

Remark 7: Our main results are based on the LMI conditions. The available Matlab YALMIP toolbox implements state-of-the-art external solvers for the actual computations. Note that, for the standard LMI system, the algorithm has a polynomial-time complexity. That is, the number $\mathcal{N}(\varepsilon)$ of flops needed to compute an $\varepsilon$-accurate solution is bounded by $O\left(\mathcal{M N}^{3} \log (\mathcal{V} / \varepsilon)\right)$, where $\mathcal{M}$ is the total row size of the LMI system, $\mathcal{N}$ is the total number of scalar decision variables, $\mathcal{V}$ is a data-dependent scaling factor, and $\varepsilon$ is relative accuracy set for algorithm. Obviously, the computational complexity of the LMI-based algorithms depends polynomially on the variable dimensions. In order to reduce the computation burden, a possible way is to conduct model reduction while preserving the main characteristics of the switched nonlinear positive systems. Fortunately, research on LMI optimization is a very active area in the applied mathematics, optimization and the operations research community, and substantial speed-ups can be expected in the future.
Remark 8: In Theorem 2, sufficient conditions on the existence of the distributed filters for switched positive systems are presented that guarantee the feasibility of the distributed filtering problem with an $l_{\infty}$ performance index. It is shown that the design problem of such filters is solvable if a feasible solution to (18) is found. In Theorem 2, the following features are considered for the underlying wireless sensor networks: (1) average $l_{\infty}$ performance index which ensures the disturbance rejection capability of the filtering dynamics; (2) missing measurements; (3) RVNs appearing in the plant under consideration; and (4) the coupling between the nodes in accordance with a topology. Hence, the distributed filters designed in Theorem 2 by utilizing the linear programming approach provides a satisfactory filtering performance against the complexities mentioned above.

\section{An Illustrative Example}

In this section, a simulation example is used to illustrate the effectiveness of the established results.

Consider the discrete-time switched positive system (1) with the following parameters:

$$
\begin{gathered}
A_{1}=\left[\begin{array}{ll}
0.32 & 0.01 \\
0.04 & 0.73
\end{array}\right], E_{1}=\left[\begin{array}{c}
0.1 \\
0
\end{array}\right], \\
G_{1}=\left[\begin{array}{c}
0.1 \\
0
\end{array}\right], M_{1}=\left[\begin{array}{cc}
0.08 & 0.11 \\
0 & 0.1
\end{array}\right]
\end{gathered}
$$

and

$$
\begin{gathered}
A_{2}=\left[\begin{array}{ll}
0.58 & 0.02 \\
0.03 & 0.67
\end{array}\right], E_{2}=\left[\begin{array}{c}
0.2 \\
0
\end{array}\right], \\
G_{2}=\left[\begin{array}{c}
0.3 \\
0
\end{array}\right], M_{2}=\left[\begin{array}{cc}
0.11 & 0.09 \\
0 & 0.15
\end{array}\right]
\end{gathered}
$$

and the RVNs are described by

$$
\begin{aligned}
& f(x(k))=x_{1} /\left(4 x_{1}^{2}+10\right)+0.2 x_{2}, \\
& g(x(k))=0.1 x_{1}+0.2 x_{2}+0.1 x_{2} \sin x_{2}, \\
& \text { Prob }\{\beta(k)=1\}=\bar{\beta}=0.85 .
\end{aligned}
$$

It is not difficult to find that the above nonlinear functions $f(x(k))$ and $g(x(k))$ satisfy (2) with

$$
\begin{gathered}
U_{1}=\left[\begin{array}{ll}
0.1 & 0.3
\end{array}\right], U_{2}=\left[\begin{array}{ll}
0 & 0.2
\end{array}\right], \\
U_{3}=\left[\begin{array}{ll}
0.15 & 0.3
\end{array}\right], U_{4}=\left[\begin{array}{ll}
0.05 & 0.1
\end{array}\right] .
\end{gathered}
$$

Suppose that there are five nodes in the sensor network represented by a directed graph $G=(\nu, \varepsilon, A)$ with

$$
\varepsilon=\left\{\begin{array}{c}
(1,1),(1,2),(1,5),(2,2),(2,3), \\
(3,3),(4,2),(4,4),(5,2),(5,5)
\end{array}\right\}
$$

which is represented by the following adjacency matrix:

$$
A=\left[\begin{array}{lllll}
1 & 1 & 0 & 0 & 1 \\
0 & 1 & 1 & 0 & 0 \\
1 & 0 & 1 & 0 & 0 \\
0 & 1 & 0 & 1 & 0 \\
0 & 1 & 0 & 0 & 1
\end{array}\right]
$$


The output of each sensor with missing measurements (7) is described by

$$
\begin{gathered}
C_{1}=\left[\begin{array}{ll}
1 & 0.2
\end{array}\right], D_{1}=0.8, C_{2}=\left[\begin{array}{ll}
0.98 & 0.1
\end{array}\right], D_{2}=1, \\
C_{3}=\left[\begin{array}{cc}
1.01 & 0.4
\end{array}\right], D_{3}=1.1, C_{4}=\left[\begin{array}{cc}
1.01 & 0.3
\end{array}\right], \\
D_{4}=0.9, C_{5}=\left[\begin{array}{ll}
1 & 0.2
\end{array}\right], D_{5}=0.7 .
\end{gathered}
$$

The probabilities of missing measurements are taken as $\bar{\gamma}_{1}=$ $0.82, \bar{\gamma}_{2}=0.92, \bar{\gamma}_{3}=0.85, \bar{\gamma}_{4}=0.78$ and $\bar{\gamma}_{5}=0.83$. According to (18) and (19) in Theorem 2, a feasible solution is found and the parameters of the distributed filters are calculated as

$$
\begin{gathered}
K_{11}=\left[\begin{array}{ll}
0.1832 & 0.1830 \\
0.1824 & 0.1822
\end{array}\right], K_{12}=\left[\begin{array}{ll}
0.1712 & 0.1712 \\
0.1705 & 0.1704
\end{array}\right], \\
K_{13}=\left[\begin{array}{ll}
0.1832 & 0.1830 \\
0.1824 & 0.1822
\end{array}\right], K_{22}=\left[\begin{array}{ll}
0.1050 & 0.1050 \\
0.1047 & 0.1047
\end{array}\right], \\
K_{23}=\left[\begin{array}{ll}
0.1123 & 0.1122 \\
0.1120 & 0.1119
\end{array}\right], K_{31}=\left[\begin{array}{ll}
0.1832 & 0.1830 \\
0.1824 & 0.1822
\end{array}\right], \\
K_{33}=\left[\begin{array}{ll}
0.1832 & 0.1830 \\
0.1824 & 0.1822
\end{array}\right], K_{42}=\left[\begin{array}{ll}
0.2530 & 0.2530 \\
0.2514 & 0.2514
\end{array}\right], \\
K_{44}=\left[\begin{array}{ll}
0.2962 & 0.2957 \\
0.2944 & 0.2938
\end{array}\right], K_{52}=\left[\begin{array}{ll}
0.1712 & 0.1712 \\
0.1705 & 0.1704
\end{array}\right], \\
K_{55}=\left[\begin{array}{ll}
0.1832 & 0.1830 \\
0.1824 & 0.1822
\end{array}\right], H_{11}=\left[\begin{array}{l}
0.0060 \\
0.0059
\end{array}\right], \\
H_{12}=\left[\begin{array}{l}
0.0048 \\
0.0048
\end{array}\right], H_{15}=\left[\begin{array}{l}
0.0059 \\
0.0059
\end{array}\right] \\
H_{22}=\left[\begin{array}{l}
0.0030 \\
0.0030
\end{array}\right], H_{23}=\left[\begin{array}{l}
0.0036 \\
0.0035
\end{array}\right] \\
H_{31}=\left[\begin{array}{l}
0.0060 \\
0.0059
\end{array}\right], H_{33}=\left[\begin{array}{l}
0.0058 \\
0.0058
\end{array}\right] \\
H_{42}=\left[\begin{array}{l}
0.0072 \\
0.0071
\end{array}\right], H_{44}=\left[\begin{array}{l}
0.0010 \\
0.0010
\end{array}\right] \\
H_{52}=\left[\begin{array}{l}
0.0048 \\
0.0048
\end{array}\right], H_{55}=\left[\begin{array}{l}
0.0059 \\
0.0059
\end{array}\right]
\end{gathered}
$$

To demonstrate the filtering performance, the form of disturbances considered is $0.1|\sin (k)|$ and the initial states $x(0)$ and $\hat{x}(0)$ are with $\left[\begin{array}{ll}6 & 8\end{array}\right]^{T}$. The switching signal is randomly generated and shown in Fig. 1. Fig. 2 indicates the state responses of switched positive systems. Fig. 3 and Fig. 4 display the output $z$ and its estimation. Fig. 5 and Fig. 6 show the estimated errors of output $z$. From Fig. 5 and Fig. 6 , it is seen that the estimated errors approach to 0 eventually. It is observed from (1), (5) and (19) that the disturbances have an impact on the design of the distributed filters by influencing on the system parameters $G_{i}$ and $D_{i}$. In order to show the corresponding estimation performance of different disturbances, Table I provides estimation performances $\alpha_{v}=$ $\sum_{i=1}^{5} \sum_{k=1}^{30} E\left\{\left\|\tilde{z}_{i}(k)\right\|_{1}\right\} /\left(5 \sum_{k=1}^{30}\|\omega(k)\|_{1}\right)$ when increasing the amplitudes of the disturbances by one, two and three times. It is seen from Table I that the estimation performances become poor with the increase of the amplitudes of the disturbances. It is observed that, although the sensor network suffers from missing measurements, the proposed distributed filter tracks the estimated output of systems quite well.
TABLE I: Different estimation performances $\alpha_{v}$.

\begin{tabular}{ccccc}
\hline Times & 0 & 1 & 2 & 3 \\
\hline$\alpha_{m}$ & 0.8493 & 0.8653 & 0.8742 & 0.8817 \\
\hline
\end{tabular}



Fig. 1: A switching signal.



Fig. 2: State responses of system (1).

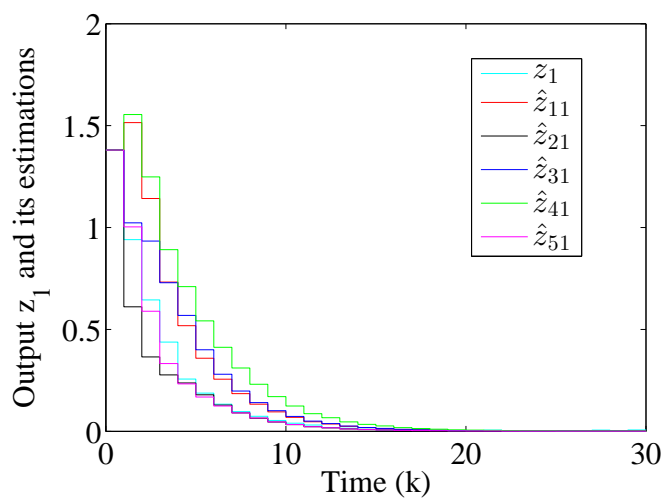

Fig. 3: Output $z_{1}$ and its estimations of system (1).

\section{CONCLUSION}

In this paper, we have analyzed the distributed filtering problem for a class of switched positive systems with stochastic nonlinearities and missing measurements over sensor networks. A novel distributed filtering technique has been proposed to satisfy the prescribed average filtering performance 


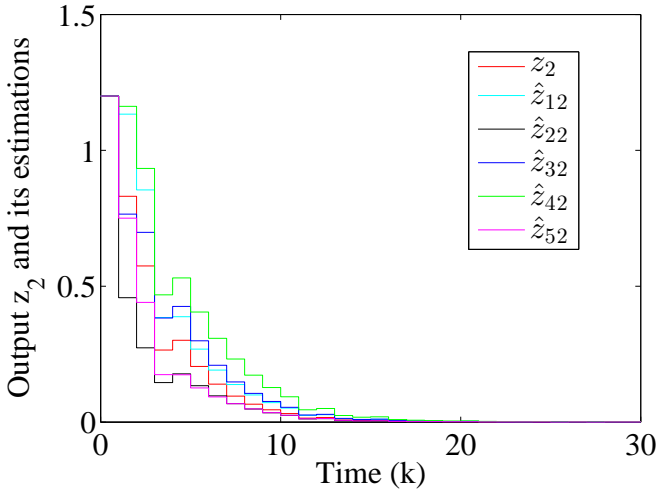

Fig. 4: Output $z_{2}$ and its estimations of system (1).



Fig. 5: Estimation errors of output $z_{1}$ of system (1).



Fig. 6: Estimation errors of output $z_{2}$ of system (1).

index. Sufficient conditions on the existence of distributed filters have been presented in terms of linear programming and the filter gains have been constructed. Finally, an illustrative example has been given to highlight the effectiveness of the developed filtering approach.

\section{REFERENCES}

[1] F. Lorenzo and R. Sergio, "Positive linear systems: theory and applications," John Wiley \& Sons, 2011.

[2] R. Shorten, F. Wirth, and D. J. Leith, "A positive systems model of tcplike congestion control: asymptotic results," IEEE/ACM Transactions on Networking, vol. 14, no. 3, pp. 616-629, 2006.
[3] Y. Zheng and G. Feng, "Two slow stabilizing switching laws for discrete time positive switched systems," International Journal of Robust and Nonlinear Control, vol. 24, pp. 2909-2927, 2014.

[4] O. Mason and R. Shorten, "On linear copositive lyapunov functions and the stability of switched positive linear systems," IEEE Transactions on Automatic Control, vol. 52, no. 7, pp. 1346-1349, 2007.

[5] J. Lian and J. Liu, "New results on stability of switched positive systems: an average dwell-time approach," IET Control Theory and Applications, vol. 7, no. 12, pp. 1651-1658, 2013.

[6] X. Liu, "Stability analysis of switched positive systems: a switched linear copositive lyapunov function method," IEEE Transactions on Circuits and Systems II: Express Briefs, vol. 56, no. 5, pp. 414-418, 2009.

[7] X. Chen, J. Lam, P. Li, and Z. Shu, " $l_{1}$-induced norm and controller synthesis of positive systems," Automatica, vol. 49, no. 5, pp. $1377-$ $1385,2013$.

[8] S. Li, Z. Xiang, and H. Karimi, "Mixed $l_{-} / l_{1}$ fault detection observer design for positive switched systems with time-varying delay via delta operator approach," International Journal of Control, Automation and Systems, vol. 12, no. 4, pp. 709-721, 2014.

[9] J. Lian, P. Shi, and Z. Feng, "Passivity and passification for a class of uncertain switched stochastic time-delay systems," IEEE Transactions on Neural Networks and Learning Systems, vol. 41, no. 1, pp. 3-13, 2013.

[10] J. Lian and J. Wang, "Passivity of switched recurrent neural networks with time-varying delays," IEEE Transactions on Neural Networks and Learning Systems, vol. 26, no. 2, pp. 357-366, 2015.

[11] Y. Liu, F. E. Alsaadi, X. Yin, and Y. Wang, "Robust $h_{\infty}$ filtering for discrete nonlinear delayed stochastic systems with missing measurements and randomly occurring nonlinearities," International Journal of General Systems, vol. 44, no. 2, pp. 169-181, 2015.

[12] D. Ding, Z. Wang, F. E. Alsaadi, and B. Shen, "Receding horizon filtering for a class of discrete time-varying nonlinear systems with multiple missing measurements," International Journal of General Systems, vol. 44, no. 2, pp. 198-211, 2015.

[13] Y. J. Liu and S. C. Tong, "Adaptive fuzzy control for a class of nonlinear discrete-time systems with backlash," IEEE Transactions on Fuzzy Systems, vol. 22, no. 5, pp. 1359-1365, 2014.

[14] Y. J. Liu, Y. Gao, S. C. Tong, and Y. M. Li, "Fuzzy approximation-based adaptive backstepping optimal control for a class of nonlinear discretetime systems with dead-zone," IEEE Transactions on Fuzzy Systems, vol. 24, no. 1, pp. 16-28, 2016.

[15] J. Liang, Z. Wang, and Y. Liu, "Distributed state estimation for discretetime sensor networks with randomly varying nonlinearities and missing measurements," IEEE Transactions on Neural Networks, vol. 22, no. 3, pp. 486-496, 2011.

[16] H. Dong, Z. Wang, and H. Gao, "Distributed filtering for a class of time-varying systems over sensor networks with quantization errors and successive packet dropouts," IEEE Transactions on Signal Processing, vol. 60, no. 6, pp. 3164-3173, 2012.

[17] D. Zhang, L. Yu, and W. Zhang, "Energy efficient distributed filtering for a class of nonlinear systems in sensor networks," IEEE Sensors Journal, vol. 15, no. 5, pp. 3026-3036, 2015.

[18] Z. Wang, Y. Wang, and Y. Liu, "Global synchronization for discrete-time stochastic complex networks with randomly occurred nonlinearities and mixed time delays," IEEE Transactions on Neural Networks, vol. 21, no. 1, pp. 11-25, 2010.

[19] Q. Liu, Z. Wang, X. He, and D. H. Zhou, "Event-based distributed filtering with stochastic measurement fading," IEEE Transactions on Industrial Informatics, vol. 11, no. 6, pp. 1643-1652, 2015.

[20] Y. Yu, H. Dong, Z. Wang, W. Ren, and F. E. Alsaadi, "Design of nonfragile state estimators for discrete time-delayed neural networks with parameter uncertainties," Neurocomputing, vol. 182, pp. 18-24, 2016.

[21] S. Mou, M. Cao, and A. S. Morse, "Target-point formation control," Automatica, vol. 61, pp. 113-118, 2015.

[22] W. Ren and R. W. Beard, "Consensus seeking in multiagent systems under dynamically changing interaction topologies," IEEE Transactions on Automatic Control, vol. 50, no. 5, pp. 655-661, 2005.

[23] S. Mou, J. Liu, and A. S. Morse, "A distributed algorithm for solving a linear algebraic equation," IEEE Transactions on Automatic Control, vol. 60, no. 11, pp. 5409-5414, 2013.

[24] H. Liu, L. Cheng, M. Tan, and Z.-G. Hou, "Containment control of continuous-time linear multi-agent systems with aperiodic sampling," Automatica, vol. 57, no. 7, pp. 78-84, 2015.

[25] C. Caione, D. Brunelli, and L. Benini, "Distributed compressive samplingfor lifetime optimization in dense wireless sensor networks," IEEE Transactions on Industrial Informatics, vol. 8, no. 1, pp. 30-40, 2012. 
[26] R. M. Estanjini, Y. Lin, and K. Li, "Optimizing warehouse for klift dispatching using a sensor network and stochastic learning," IEEE Transactions on Industrial Informatics, vol. 7, no. 3, pp. 476-486, 2011.

[27] H. Dong, Z. Wang, F. E. Alsaadi, and B. Ahmad, "Event-triggered robust distributed state estimation for sensor networks with state-dependent noises," International Journal of General Systems, vol. 44, no. 2, pp. 254-266, 2015.

[28] X. Su, L. Wu, and P. Shi, "Sensor networks with random link failures: distributed filtering for t-s fuzzy systems," IEEE Transactions on Industrial Informatics, vol. 9, no. 3, pp. 1739-1750, 2013.

[29] J. Zhang, Z. Wang, D. Ding, and X. Liu, " $h_{\infty}$ state estimation for discrete-time delayed neural networks with randomly occurring quantizations and missing measurements," Neurocomputing, vol. 148, pp. 388-396, 2015

[30] N. Hou, H. Dong, Z. Wang, W. Ren, and F. E. Alsaadi, "Nonfragile state estimation for discrete markovian jumping neural networks," Neurocomputing, vol. 179, pp. 238-245, 2016.

[31] H. Yu, Y. Liu, and W. Wang, "Distributed sparse signal estimation in sensor networks using $h_{\infty}$-consensus filtering," IEEE/CAA Journal of Automatica Sinica, vol. 1, no. 2, pp. 149-154, 2014.

[32] W. Yu, G. Chen, and Z. Wang, "Distributed consensus filtering in sensor networks," IEEE Transactions on Systems Man and Cybernetics: Part B Cybernetics, vol. 39, no. 6, pp. 1568-1577, 2009.

[33] M. Basin, S. ElviraCeja, and E. Sanchez, "Mean-square $h_{\infty}$ filtering for stochastic systems: application to a 2dof helicopter," Signal Processing, vol. 92 , no. 3, pp. 801-806, 2012.

[34] R. Caballeroguila, A. Hermosocarazo, and J. Linaresprez, "Optimal state estimation for networked systems with random parameter matrices, correlated noises and delayed measurements," International Journal of General Systems, vol. 44, no. 2, pp. 142-154, 2015.

[35] M. Chadli and H. R. Karimi, "Robust observer design for unknown inputs takagi-sugeno models," IEEE Transactions on Fuzzy Systems, vol. 21, no. 1, pp. 158-164, 2013.

[36] H. R. Karimi, "Robust $h_{\infty}$ filter design for uncertain linear systems over network with network-induced delays and output quantization," Model, Identification and Control, vol. 30, no. 1, pp. 27-37, 2009.

[37] L. Wu and C. Ho, "Reduced-order $l_{2}-l_{\infty}$ filtering of switched nonlinear stochastic systems," IET Control Theory and Applications, vol. 3, no. 5, pp. 493-508, 2009.

[38] H. Gao and T. Chen, " $h_{\infty}$ estimation for uncertain systems with limited communication capacity," IEEE Transactions on Signal Processing, vol. 52, no. 11, pp. 2070-2084, 2007.

[39] B. Shen, Z. Wang, and Y. S. Hung, "Distributed consensus $h_{\infty}$ filtering in sensor networks with multiple missing measurements: The finitehorizon case," Automatica, vol. 46, no. 10, pp. 1682-1688, 2010.

[40] Z. Wu, J. Park, H. Su, and J. Chu, "Non-fragile synchronisation control for complex networks with missing data," International Journal of Control, vol. 86, no. 3, pp. 555-566, 2013.

[41] Y. Luo, Z. Wang, G. Wei, and F. E. Alsaadi, "Robust $h_{\infty}$ filtering for a class of two-dimensional uncertain fuzzy systems with randomly occurring mixed delays," IEEE Transactions on Fuzzy Systems, to be published,2016.

[42] L. Wang, Z. Wang, T. Huang, and G. Wei, "An event-triggered approach to state estimation for a class of complex networks with mixed time delays and nonlinearities," IEEE Transactions on Cybernetics, to be published, 2016 